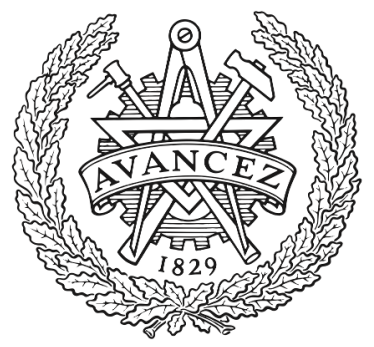

CHALMERS

UNIVERSITY OF TECHNOLOGY

\title{
Gain of electron orbital angular momentum in a direct laser acceleration process
}

Downloaded from: https://research.chalmers.se, 2023-04-26 12:46 UTC

Citation for the original published paper (version of record):

Nuter, R., Korneev, P., Dmitriev, E. et al (2020). Gain of electron orbital angular momentum in a direct laser acceleration process. PHYSICAL REVIEW E, 101(5).

http://dx.doi.org/10.1103/PhysRevE.101.053202

N.B. When citing this work, cite the original published paper. 


\title{
Gain of electron orbital angular momentum in a direct laser acceleration process
}

\author{
R. Nuter, ${ }^{1}$ Ph. Korneev $\odot,{ }^{2,3}$ E. Dmitriev, ${ }^{2}$ I. Thiele, ${ }^{4}$ and V. T. Tikhonchuk $\oplus^{1,5}$ \\ ${ }^{1}$ Université de Bordeaux, CNRS, CEA, UMR 5107, 33405 Talence, France \\ ${ }^{2}$ National Research Nuclear University “MEPhI”, Moscow 115409, Russian Federation \\ ${ }^{3}$ P.N. Lebedev Physical Institute, Moscow 119333, Russian Federation \\ ${ }^{4}$ Department of Physics, Chalmers University of Technology, SE-41296 Göteborg, Sweden \\ ${ }^{5}$ ELI-Beamlines, Institute of Physics, Czech Academy of Sciences, 25241 Dolni Brezany, Czech Republic
}

(Received 12 November 2019; revised manuscript received 27 January 2020; accepted 9 April 2020; published 6 May 2020)

\begin{abstract}
Three-dimensional "particle in cell" simulations show that a quasistatic magnetic field can be generated in a plasma irradiated by a linearly polarized Laguerre-Gauss beam with a nonzero orbital angular momentum (OAM). Perturbative analysis of the electron dynamics in the low intensity limit and detailed numerical analysis predict a laser to electrons OAM transfer. Plasma electrons gain angular velocity thanks to the dephasing process induced by the combined action of the ponderomotive force and the laser induced-radial oscillation. Similar to the "direct laser acceleration," where Gaussian laser beams transmit part of its axial momentum to electrons, Laguerre-Gaussian beams transfer a part of their orbital angular momentum to electrons through the dephasing process.
\end{abstract}

DOI: 10.1103/PhysRevE.101.053202

\section{INTRODUCTION}

The recent advent of laser sources carrying orbital angular momentum in the relativistic intensity regime contribute to an increasing interest to their interaction with plasma. These works are motivated by numerous applications such as highorder harmonic generation with controlled orbital angular momentum (OAM) [1,2], amplification of twisted laser pulses via stimulated Raman scattering [3,4] particle acceleration [5], and magnetic field generation [6-9]. Several numerical configurations have been proposed to generate a quasistatic magnetic field. Ali et al. [6] theoretically propose to replace the usually considered circularly polarized Gaussian beam in the inverse Faraday effect by a linearly polarized LaguerreGaussian (LG) beam [10]. In their numerical setup, the generated azimuthal current requires the bremstrahlung process to trigger the energy transfer from laser to electrons. Shi et al. [7] numerically demonstrate that the magnetic field can be generated by plasma waves driven by two copropagating intense twisted lasers. All of these works require an intermediate process to transfer OAM from laser to electrons. A direct transfer is studied in Ref. [8], where Hu et al. propose to irradiate a wire target with a relativistic Laguerre-Gaussian pulse and, in Ref. [9], where we theoretically and numerically demonstrate that a wireless solenoid could be produced from the irradiation of a low dense plasma with radially polarized LG beams. Beyond all of these works, there is still no answer on what is the electron dynamics when a homogeneous low dense plasma is irradiated by relativistic linearly polarized LG beams. In this simplest possible configuration, does the laser beam transfer OAM to electrons, and what is the associated electron OAM distribution?

This work aims to describe this plasma dynamics. First, in Sec. II, we focus our attention on the dynamics of plasma electrons experiencing only the action of an OAM laser beam. We do not consider the influence of the self-consistent fields generated by the plasma. In Sec. II A, a perturbative approach is applied to the motion equations for electrons in order to identify the accelerating processes. It requires analytical expressions for the electromagnetic field components, which are computed from an expansion of the laser OAM fields over the LG basis in Appendix B. The second order approximation considered in the perturbative analysis is appropriate for the low intensity regime. It is not sufficient to explain the laser to plasma OAM transfer observed at high laser intensity. A detailed numerical analysis of PIC simulations, performed in Sec. II B, reveals that similar to the "direct laser acceleration" mechanism [11,12], where Gaussian laser beam transmits a part of its axial momentum to electrons when a dephasing process breaks the adiabaticity between the laser pulse and the electrons, LG beam transfers a part of its OAM to electrons through the dephasing action of the ponderomotive force combined with the laser-induced radial oscillation. Finally, we show in Sec. III that the plasma induced self-consistent fields reduce the efficiency of the laser to electrons OAM transfer. However, for the chosen laser and plasma parameters, we observe the generation of a quasistatic magnetic field, which survives over more than $50 \mathrm{fs}$.

\section{DYNAMICS OF PLASMA ELECTRONS DRIVEN BY A LAGUERRE-GAUSS BEAM-NO PLASMA-INDUCED SELF-CONSISTENT FIELDS}

In a first approach, we focus our attention on the dynamics of electrons in a linearly polarized laser beam carrying an OAM. We do not consider the effect of plasma-induced selfconsistent fields. This last feature will be analyzed further, in Sec. III. 
At the focal point $x=x_{f}$, a linearly polarized OAM laser beam is defined in the cylindrical coordinates $(r, \theta, x)$ with

$$
\vec{E}\left(r, \theta, t, x_{f}\right)=E_{0} g(t)\left(\frac{r}{w_{0}}\right)^{|l|} e^{-\left(\frac{r}{w_{0}}\right)^{2}} \cos \left(l \theta-\omega_{0} t\right) \vec{e}_{y},
$$

with the temporal envelope $g(t)=\cos ^{2}\left(\pi \frac{t-t_{0}}{\tau}\right)$ in the time interval $\left|t-t_{0}\right|<\tau / 2$, the laser OAM index $l$, the focal beam waist $w_{0}$ equal to 2.5 laser wavelength, the pulse duration $\tau$ equal to $n=6$ optical periods $\left(T=2 \pi / \omega_{0}\right)$, the central beam time $t_{0}$, the laser amplitude $E_{0}$, and the $y$-unit vector $\vec{e}_{y}$. In practical applications, one may consider the laser frequency $\omega_{0}=2.3 \times 10^{15} \mathrm{~s}^{-1}$ corresponding to the laser wavelength $\lambda_{0}=0.8 \mu \mathrm{m}$. In the following, we express the physical quantities in dimensionless units as presented in Appendix A.

\section{A. Analytical development of electron dynamics}

The relativistic equations of motion for the electrons are written in the dimensionless units

$$
\begin{aligned}
& \dot{p_{r}}=-E_{r}-v_{\theta} B_{x}+v_{x} B_{\theta}+p_{\theta} \dot{\theta}, \\
& \dot{p_{\theta}}=-E_{\theta}-v_{x} B_{r}+v_{r} B_{x}-p_{r} \dot{\theta}, \\
& \dot{p}_{x}=-E_{x}-v_{r} B_{\theta}+v_{\theta} B_{r},
\end{aligned}
$$

where $p_{r}=\gamma v_{r}=\gamma \dot{r}, p_{\theta}=\gamma v_{\theta}=\gamma r \dot{\theta}$, and $p_{x}=\gamma v_{x}=$ $\dot{x}$ are the radial, azimuthal, and longitudinal electron momenta, respectively, $\gamma=\sqrt{1+p_{r}^{2}+p_{\theta}^{2}+p_{x}^{2}}$ is the Lorentz factor, and $r, \theta, x$ are the cylindrical coordinates. Here, the $E_{r}, E_{\theta}, E_{x}, B_{r}, B_{\theta}$, and $B_{x}$ describe only the electromagnetic field components; no plasma self-consistent fields are considered:

$$
\begin{aligned}
& E_{r}(r, \theta, x)=a_{0} f(r) g(t) \cos \left[t-l \theta-\left(x-x_{f}\right)\right] \cos \theta \\
& E_{\theta}(r, \theta, x)=-a_{0} f(r) g(t) \cos \left[t-l \theta-\left(x-x_{f}\right)\right] \sin \theta \\
& E_{x}(r, \theta, x)= a_{0} \frac{f(r)}{r} g(t)\left\{\left[|l|-2\left(\frac{r}{w_{0}}\right)^{2}\right]\right. \\
& \times \cos \theta \sin \left[t-l \theta-\left(x-x_{f}\right)\right] \\
&+\left.l \sin \theta \cos \left[t-l \theta-\left(x-x_{f}\right)\right]\right\} \\
& B_{r}(r, \theta, x)=-E_{\theta}, \\
& B_{\theta}(r, \theta, x)=E_{r}, \\
& B_{x}(r, \theta, x)= a_{0} \frac{f(r)}{r} g(t)\left\{\left[l \mid-2\left(\frac{r}{w_{0}}\right)^{2}\right]\right. \\
& \times \sin \theta \sin \left[t-l \theta-\left(x-x_{f}\right)\right] \\
&\left.-l \cos \theta \cos \left[t-l \theta-\left(x-x_{f}\right)\right]\right\}
\end{aligned}
$$

where $a_{0}=\frac{e E_{0}}{m_{e} \omega_{0} c}$ is the dimensionless laser field amplitude, $m_{e}, e$ are the mass and charge of electron, respectively, $c$ is the light velocity in vacuum, and $f(r)=\left(r / w_{0}\right)^{|l|} e^{-\left(r / w_{0}\right)^{2}}$ is the beam radial distribution. The computations of the OAM laser field components are detailed in Appendix B. Equations (5)-(10) have been obtained by neglecting the Gouy phase, its integration over $x$, and the curvature terms because the laser-plasma interaction takes place over a longitudinal displacement smaller than the Rayleigh length $\left(\left|x-x_{f}\right|<x_{R}\right)$.

In a low laser intensity regime, we apply a perturbative analysis on the equations of motion Eqs. (2)-(4) and calculate the electron OAM value $L_{x}=r p_{\theta}$. The perturbative development is expressed in powers of $a_{0}$, such that the physical quantities are written as $X=X^{(0)}+X^{(1)}+X^{(2)}+\cdots$ with $X^{(i)}$ depending on $a_{0}^{i}$, where $i$ denotes the power order. At the first order, the equation for the electron OAM reads $d_{t} L_{x}^{(1)}=$ $r_{0} d_{t} p_{\theta}^{(1)}$, where $r_{0}, \theta_{0}, x_{0}$ are the electron initial coordinates, $d_{t} p_{\theta}^{(1)}=-E_{\theta}$, and $d_{t}=\frac{d}{d t}$ denotes the time derivative. This results in

$$
\begin{aligned}
L_{x}^{(1)}(t)= & \frac{a_{0}}{2} r_{0} f\left(r_{0}\right) \sin \theta_{0} \\
& \times\left[\sin \left(t-\phi_{0}\right)+\frac{1}{2\left(1+\frac{1}{n}\right)} \sin \left(\left[1+\frac{1}{n}\right] t-\phi_{0}\right)\right. \\
& +\frac{1}{2\left(1-\frac{1}{n}\right)} \sin \left(\left[1-\frac{1}{n}\right] t-\phi_{0}\right) \\
& \left.-(-1)^{n} \frac{1}{n^{2}-1} \sin \phi_{0}\right]
\end{aligned}
$$

with $n=6$ the number of optical periods in the pulse duration and $\phi_{0}=l \theta_{0}+x_{0}-x_{f}$. By integrating Eq. (11) over the laser pulse duration and by averaging it over the initial longitudinal positions, we obtain $L_{x}^{(1)}(n T)=0$. We conclude that, in the first order, no laser OAM is transferred to electron.

Thus we compute the electron OAM at the second order. Its time derivative solves

$$
d_{t} L_{x}^{(2)}=r^{(1)} d_{t} p_{\theta}^{(1)}+p_{r}^{(1)} p_{\theta}^{(1)}+r_{0} d_{t} p_{\theta}^{(2)},
$$

where $d_{t} p_{\theta}^{(2)}$ is the second order of Eq. (3):

$$
d_{t} p_{\theta}^{(2)}=-\frac{d E_{\theta}}{d \vec{r}} \vec{r}^{(1)}-p_{x}^{(1)} B_{r}+p_{r}^{(1)} B_{x}-\frac{p_{r}^{(1)} p_{\theta}^{(1)}}{r_{0}} .
$$

It results in

$$
d_{t} L_{x}^{(2)}=r^{(1)} d_{t} p_{\theta}^{(1)}-r_{0} p_{x}^{(1)} B_{r}+r_{0} p_{r}^{(1)} B_{x}-r_{0} \frac{d E_{\theta}}{d \vec{r}} \vec{r}^{(1)},
$$

where the electron momenta and coordinates solve

$$
\begin{gathered}
d_{t} p_{r}^{(1)}=-E_{r}, \quad d_{t} r^{(1)}=p_{r}^{(1)}, \\
d_{t} p_{\theta}^{(1)}=-E_{\theta}, \quad d_{t} \theta^{(1)}=\frac{p_{\theta}^{(1)}}{r_{0}} \\
d_{t} p_{x}^{(1)}=-E_{x}, \quad d_{t} x^{(1)}=p_{x}^{(1)} .
\end{gathered}
$$

By including solutions of Eq. (15) (given in Appendix C) in Eq. (14), and averaging over initial longitudinal positions, we obtain

$$
L_{x}^{(2)}(n T)=n \pi\left(\frac{a_{0} f\left(r_{0}\right)}{2}\right)^{2}\left[|l|-2\left(\frac{r_{0}}{w_{0}}\right)^{2}\right] \frac{\frac{1}{n^{2}}}{\left(1-\frac{1}{n^{2}}\right)^{2}} \sin 2 \theta
$$

Thus the perturbative theory predicts an OAM transfer from the laser beam to the electrons. However, the presence of a $\sin 2 \theta$ term in the $L_{x}^{(2)}(n T)$ expression sets to zero the final angle-integrated plasma OAM. Opposite to the radially 


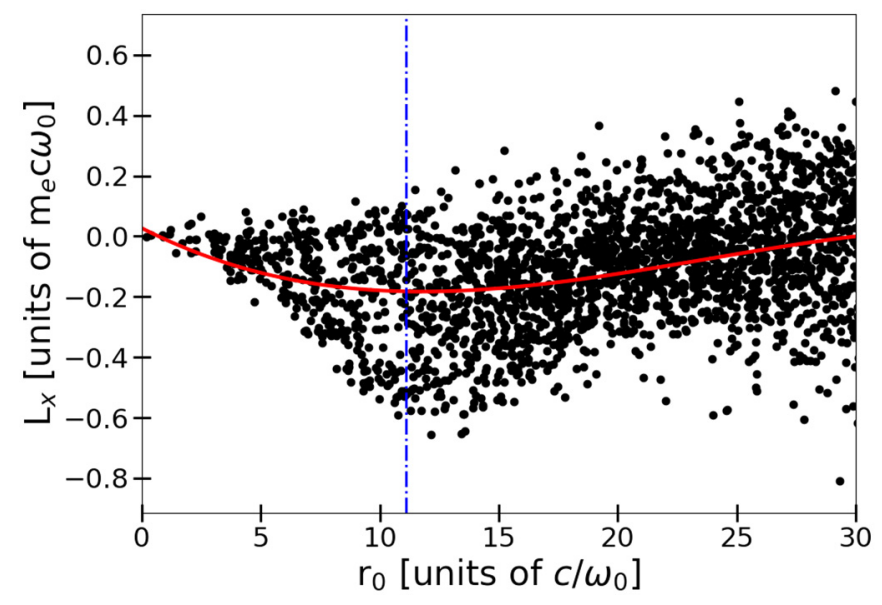

FIG. 1. Electron OAM distribution versus the initial radial coordinate computed at $\omega_{0} t=150$ (black circle) once the laser has left the plasma. The red solid curve draws the averaged value; the blue dotted curve displays the radial position where the laser intensity is maximal.

polarized case [9], where the perturbative theory predicts a magnetic field generation, the linearly polarized case enables a laser to electron OAM transfer, but no quasistatic magnetic field generation because the averaged electron OAM over the azimuthal angle is zero.

\section{B. Numerical modeling of the electron dynamics}

The interaction of a linearly polarized OAM laser beam with a collisionless underdense plasma is studied with the 3D PIC code OCEAN [13]. To compare with the analytical development, we switch off the computation of the plasma induced self-consistent fields in the PIC code such that plasma particles only experience the electric and magnetic fields of the laser beam. Thus the laser beam propagation is not affected by the presence of plasma. The numerical box is composed of 1000 cells along the longitudinal axis $x$, and $804 \times 804$ cells in the transverse plane $(y, z)$, with a spatial resolution of $\lambda_{0} / 42$. Absorbing conditions for the electromagnetic fields and particles are defined at the box boundaries. The laser pulse is characterized with an OAM $l=1$ and an intensity $2 \times 10^{18} \mathrm{~W} / \mathrm{cm}^{2}$ at the focal plane, $x_{f}=8 \mu \mathrm{m}$. It is injected into the numerical box from the left border by considering Maxwell's consistent algorithm described in [14]. The total laser energy is $2.5 \mathrm{~mJ}$. The plasma composed of electrons and protons has an initial density equal to $1.74 \times$ $10^{19} \mathrm{~cm}^{-3}$, corresponding to $1 \%$ of the plasma critical density $n_{c}=1.74 \times 10^{21} \mathrm{~cm}^{-3}$. Localized at the left border of the numerical box, it has a rectangular shape filling the transverse dimension of the box and a length equal to 13 wavelengths. With these parameters, the laser-plasma interaction takes place from $\omega_{0} t=0$ to $\omega_{0} t=135$. Ten macroparticles per cell are considered, and the macroparticles are initialized with a zero velocity.

Black circles in Fig. 1 display radial distribution of the electron orbital angular momentum once the laser field has left the plasma $\left(\omega_{0} t=150\right)$. Note that, for computational reasons, we could not store the characteristics of all plasma electrons,

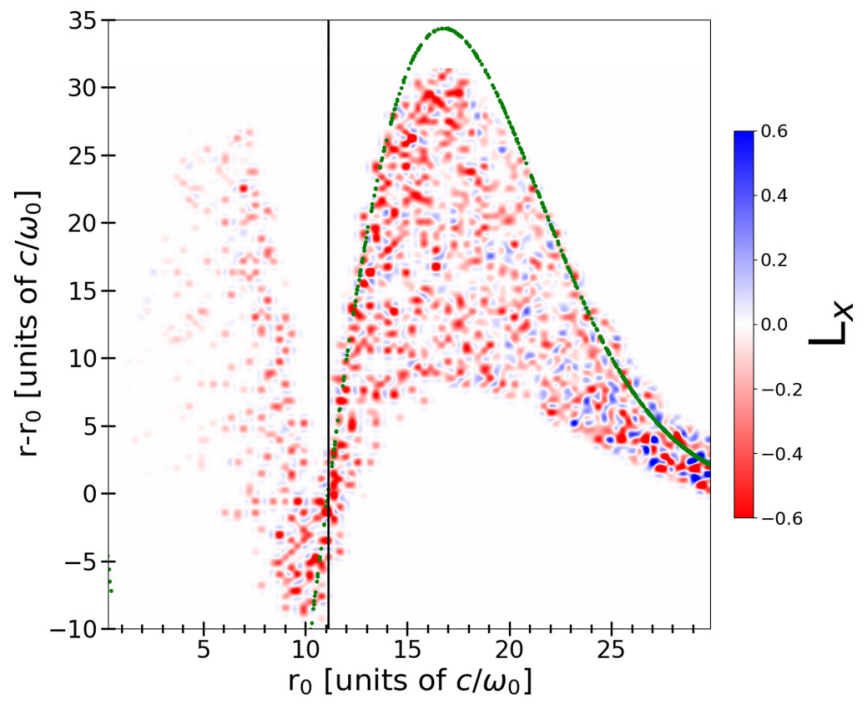

FIG. 2. Distribution of the electron $L_{x}$ computed at $\omega_{0} t=150$ versus the radial displacement $r-r_{0}$ and the initial radial coordinate $r_{0}$ once the laser left the plasma (data have been interpolated with a spline function). Green dotted curve displays electron radial displacement induced by the radial ponderomotive force. Black solid curve shows the radial position where the laser intensity is maximal.

only a few of them are tracked and displayed. These tracked particles have been randomly chosen in the whole plasma area. As predicted by the perturbative theory, we observe a laser to electrons OAM transfer. Whereas this transfer is inefficient near the axis $(r=0)$, electrons gain higher OAM as their initial radial coordinate increases. The red solid curve, showing the averaged $L_{x}$ value, highlights nonzero values for an initial radial coordinate smaller than $25 c / \omega_{0}$. This is different from the perturbative theory result, which predicts a zero angle-averaged electron OAM. We observe that the transfer is maximal for electrons initially localized near the laser peak intensity (shown with the blue dotted curve).

Because the transverse spatial distribution of the laser beam is inhomogeneous (characterized with a donut shape) electrons gain an average momentum due to the ponderomotive force:

$$
\left\langle\vec{p}^{(2)}\right\rangle=-\frac{a_{0}^{2}}{4} \int_{0}^{t} \vec{\nabla}\left[f^{2}\left(r_{0}\right) g^{2}\left(t^{\prime}\right)\right] d t^{\prime},
$$

where $f(r)$ is the radial function defined in Sec. II A and $g(t)$ is the temporal envelope given in Eq. (1). By computing the radial component from Eq. (17), we obtain

$$
\left\langle p_{r}^{(2)}\right\rangle=-\frac{a_{0}^{2}}{4} f^{2}\left(r_{0}\right)\left[|l|-2\left(\frac{r_{0}}{w_{0}}\right)^{2}\right] \frac{t}{r_{0}}
$$

resulting in the radial displacement

$$
\Delta r=-\frac{a_{0}^{2}}{4} \frac{f^{2}\left(r_{0}\right)}{r_{0}}\left[|l|-2\left(\frac{r_{0}}{w_{0}}\right)^{2}\right] t^{2},
$$

where $\left\langle p^{(2)}\right\rangle, \Delta r$, and $t$ are expressed in units of $m_{e} c, c / \omega_{0}$, and $1 / \omega_{0}$, respectively.

Figure 2(a) details the dynamics by displaying the electron $L_{x}$ versus the initial radial position $\left(r_{0}\right)$ and their final displacement $\Delta r=r-r_{0}$ at $\omega_{0} t=150$. The green dotted curve 


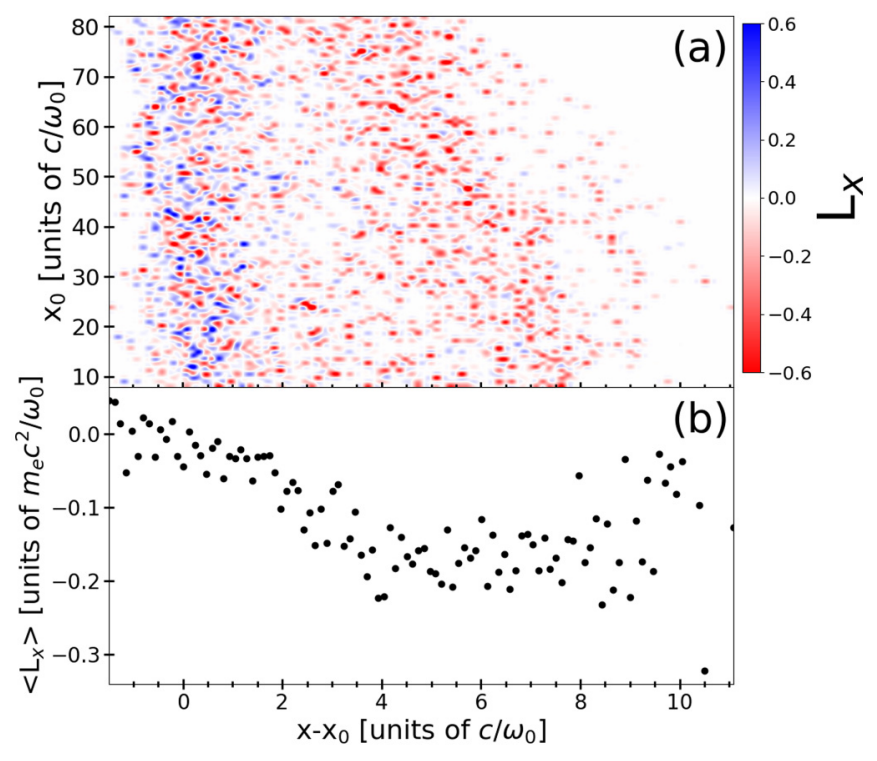

FIG. 3. Distribution of the electron $L_{x}$ computed at $\omega_{0} t=150$ versus the longitudinal displacement $x-x_{0}$ and the initial longitudinal coordinate $x_{0}$ (a) once the laser left the plasma area (data have been interpolated with a spline function). Averaged value of $L_{x}$ versus $x-x_{0}$ (b).

shows the electron radial displacement due to the ponderomotive force [Eq. (19)], when the interaction with the laser is ended. This curve fits perfectly the electron $L_{x}$ distribution from the PIC simulations, and confirms that electrons experience a radial drift due to the laser induced ponderomotive force. However, we observe that the electron radial displacement does not influence the final OAM values. Indeed, the maximal amplitudes of $L_{x}$ are obtained in the $8 c / \omega_{0}<r_{0}<$ $18 c / \omega_{0}$ zone, whatever the final radial displacement is. It is ever more intriguing that electrons gain their maximal OAM where the ponderomotive force is zero $(\Delta r=0)$. We then conclude that the constant part of laser-induced radial ponderomotive force does not contribute to the laser to plasma OAM transfer.

Figure 3(a) shows the $L_{x}$ distribution as a function of their longitudinal displacement $\left(x-x_{0}\right)$ and their initial longitudinal coordinate $x_{0}$. Electrons that do not experience longitudinal displacement $\left(x-x_{0}<\lambda_{0} / 3\right)$ gain positive or negative $L_{x}$ values randomly. These electrons do not contribute to the nonzero plasma $L_{x}$ value. However, those ones which move over a higher longitudinal distance gain mainly negative $L_{x}$ values.

Figure 3(b) displays the averaged electron $L_{x}$ values as a function of the longitudinal displacement. We observe an increase of the $L_{x}$ amplitude with the longitudinal displacement up to a saturation value that is reached for a longitudinal displacement equal to one laser wavelength. It appears evident that the laser to plasma OAM transfer is made possible due to the longitudinal displacement of the electrons. This axial displacement, caused by the longitudinal ponderomotive force, is not accounted for in the second order perturbative development of $L_{x}$; it requires the development to higher order terms. This momentum transfer from laser to electron appears very similar to the so-called "direct laser acceleration" (DLA) [11,12]. The constant part of the ponderomotive force accelerates longitudinally the electrons and breaks the adiabaticity between the laser and the electrons. The field seen by a moving electron appears chirped, which results in a longitudinal and angular momentum gain. The following part of the manuscript details this feature.

\section{Dependence of the OAM transfer on the laser polarization}

Using the PIC code, we also model the interaction of azimuthally and radially polarized LG beams with a low dense plasma. The simulation with the azimuthally polarized LG beam is the one which displays the less efficient laser to plasma OAM transfer. The final $L_{x}$ values are much weaker than in the linearly polarized case. By contrast, as demonstrated in Ref. [9], the radially polarized OAM laser beam leads to the most efficient laser to plasma OAM transfer.

We consider these three polarization cases to compare the dynamics of a single electron interacting with an OAM laser beam. This electron has an initial radial coordinate in the spatial region where the laser field is maximal. Figure 4 displays the time evolution of four temporal diagnostics for three different laser polarizations (radial, azimuthal, and linear). Figure 4(a), displaying the electron $L_{x}$ temporal evolution, shows the final $L_{x}$ values equal to $0.03 m_{e} c \omega_{0} / e$ for the azimuthally polarized LG beam, $-0.45 m_{e} c \omega_{0} / e$ for the linearly polarized LG beam, and $-0.55 m_{e} c \omega_{0} / e$ for the radially polarized one. The laser to electron OAM transfer efficiency is higher for the radial polarization, as already shown in [9].

The electron in the three polarization cases display almost the same longitudinal displacement [Fig. 4(c)], due to the constant part of the longitudinal ponderomotive force. This force, originating from the temporal shape of the laser beam, is similar for the three polarization cases. The main difference in the particle dynamics is observed in the radial motion [Fig. 4(b)]. Because of the weak radial component for the azimuthally polarized beam, the particle experiences a very weak radial oscillation. In contrast, these oscillations are larger for the linear and radial polarizations. The laser to electron OAM transfer is efficient thanks to these forced radial oscillations combined with the longitudinal motion of the electrons. The constant part of the longitudinal ponderomotive force accelerates electrons, which results in an electron longitudinal momentum gain. Because of this longitudinal velocity, electrons do not experience a periodically oscillating radial field $\left[E_{r} \simeq \sin (t)\right]$, but a "temporally chirped" one $\left[E_{r} \simeq \sin \left(t-\alpha t^{2}\right)\right]$. This is observable in Figs. 4(b) and $4(\mathrm{c})$, where the radial position displays a temporal oscillation with an increasing period. This process, which breaks the adiabaticity between the laser and the electron, is similar to the dephasing process in the direct laser acceleration mechanism, where an additional electrostatic field is added to the interaction $[15,16]$. Then, electrons experience a large radial oscillation and gain an angular velocity in the field oscillation $\left(p_{\theta} \simeq \frac{d E_{\theta}}{d r}\right)$.

The axial momentum transfer from a Gaussian beam to electrons in the DLA process is made possible because of the longitudinal acceleration. Here, we show that the laser does 

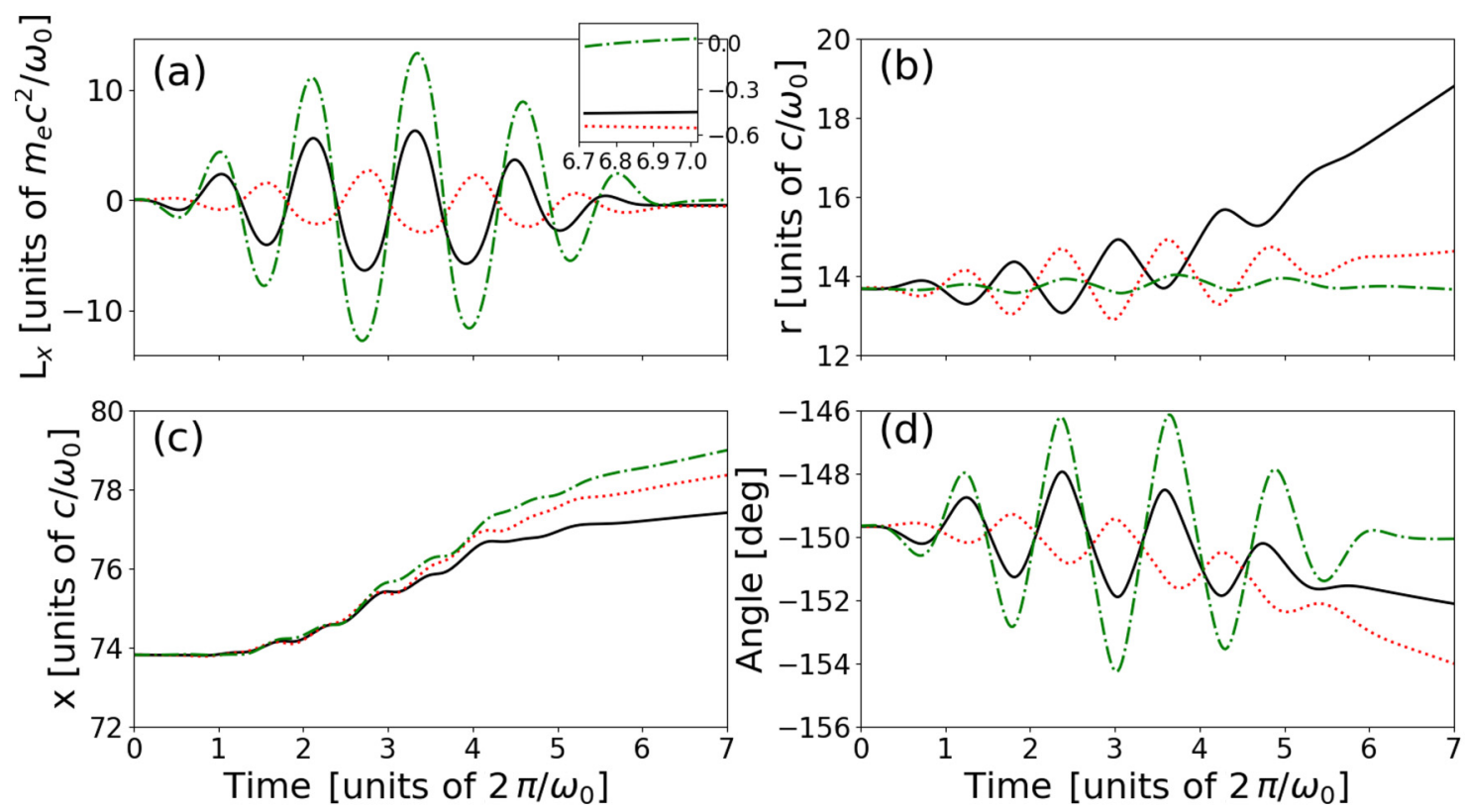

FIG. 4. Temporal evolution of (a) orbital angular momentum, (b) radial coordinate, (c) longitudinal position, and (d) azimuthal angle of a single "tracking particle" for three laser polarizations: linear (black solid curve), radial (red dotted curve), and azimuthal (green dash-dotted curve). The inset figure in (a) displays a zoom of the panel (a), i.e. the $L_{x}$ values as a function of the time expressed in units of $2 \pi / \omega_{0}$.

not transfer only its axial momentum; it can also transfer part of its orbital angular momentum if it is nonzero.

\section{DYNAMICS OF ELECTRONS PLASMA IRRADIATED BY A LAGUERRE-GAUSS BEAM AND EXPERIENCING THE INDUCED SELF-CONSISTENT FIELDS}

In the previous section, we have identified the physical processes which make the laser to plasma OAM transfer possible. This identification has been performed by neglecting the plasma induced self-consistent fields. Now, we present PIC simulations where the plasma self-consistent fields are switched on, so that the plasma electrons experience OAM laser fields modified by the plasma induced self-consistent fields.

\section{A. Laser to plasma OAM transfer}

Figure 5 compares the electron $L_{x}$ radial distribution from PIC simulations where plasma induced self-consistent fields are activated (yellow triangles, red dotted curve) and deactivated (black circles, red curve). As in Sec. II B, we observe a nonzero averaged plasma $L_{x}$ value, resulting from an inhomogeneous azimuthal distribution. However, the plasma self-consistent fields reduce the amplitude of plasma electron OAM values. Whereas, at the maximum peak intensity, the plasma OAM reaches $-0.2 m_{e} c \omega_{0} / e$ when the plasma induced self-consistent fields are switched off, it only reaches $-0.18 m_{e} c \omega_{0} / e$ when they are considered.

The plasma, defined with a finite longitudinal length, possesses charge separating fields on its border [17]. These electrostatic fields keep the electrons trapped in the plasma area, such that their interaction with the laser field is reduced. As a result, the electron OAM values are also reduced but not canceled, such that, for realistic laser and plasma parameters, the laser OAM may be transferred to the plasma.

Figure 6(a) presents the transverse spatial distribution of the electron $L_{x}$. In the central part of the distribution $(r \simeq$ $\left.11.6 c / \omega_{0}\right)$, we observe two lobes with positive values and two lobes with negative values, as predicted by the perturbative theory [near $\sin (2 \theta)]$. But, in contrast to this theory, their respective weights are not equal and we note a large prevalence of electrons with negative OAM. This results in a nonzero value for the plasma OAM displayed in Fig. 5 with the red curve.

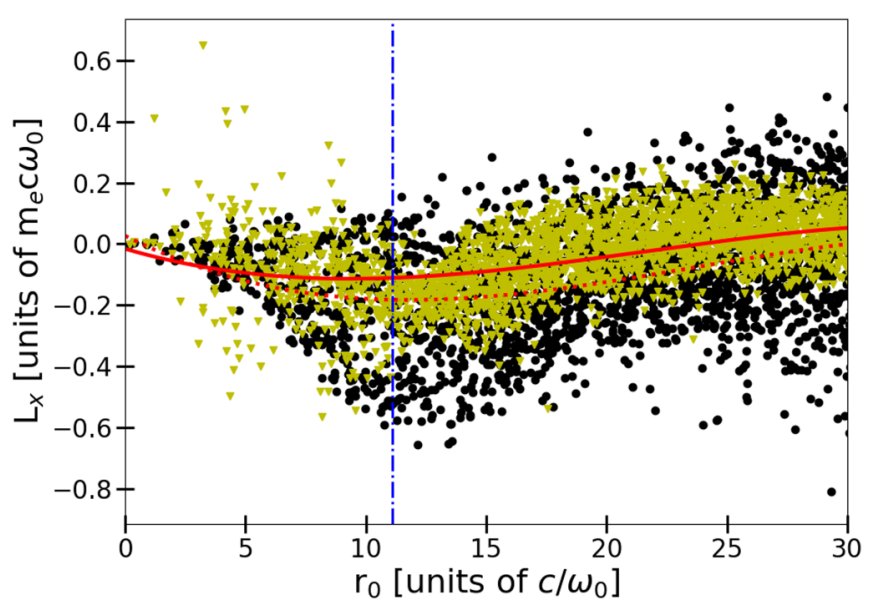

FIG. 5. Electron $L_{x}$ radial distribution when the self-consistent fields are switched on (yellow triangles) or switched off (black circles) at $\omega_{0} t=150$, once the laser left the plasma. The corresponding plasma averaged $L_{x}$ values are displayed with red and red dotted curve, respectively. 

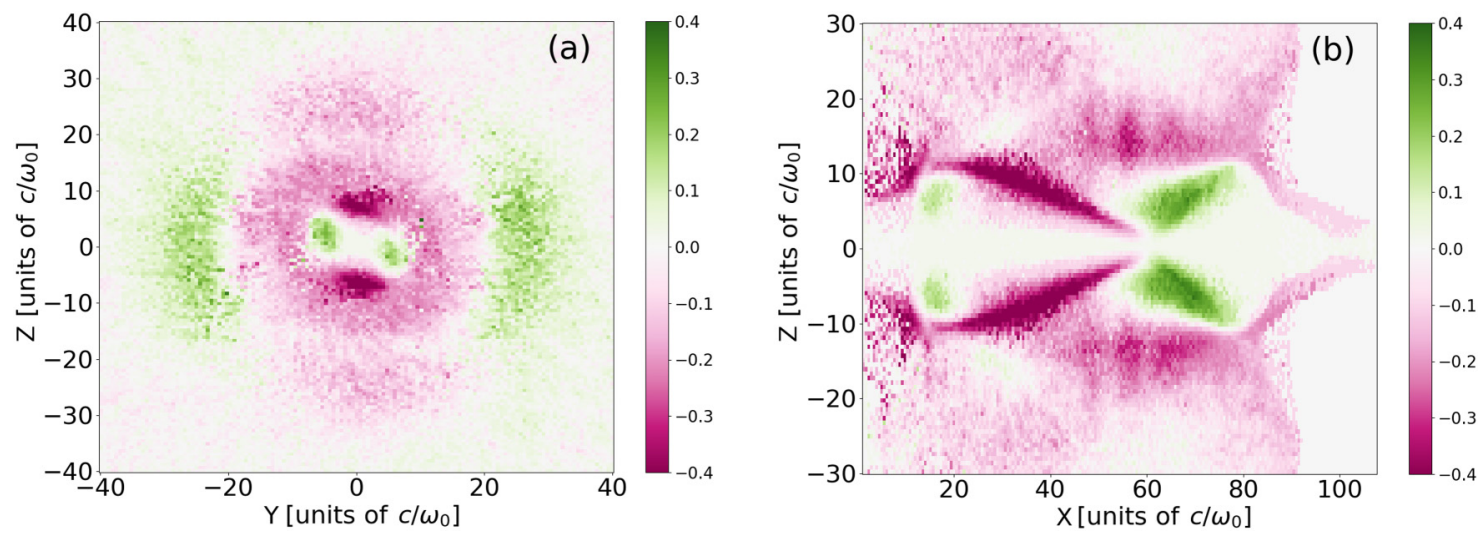

FIG. 6. (a) Transverse and (b) longitudinal spatial distribution of the electron OAM at $\omega_{0} t=165$ computed at $x_{0}=40 \mathrm{c} / \omega_{0}$ and $y_{0}=0$, respectively, once the laser beam left the plasma.

The longitudinal distribution of $L_{x}$ in Fig. 6(b) shows that the laser transfers part of its OAM to all the plasma electrons. This transfer is optimal at a radius corresponding to the laser peak intensity. The focal point, localized at $x_{f}=62.83 \mathrm{c} / \omega_{0}$, separates negative from positive $L_{x}$ values.

\section{B. Magnetic field}

We compare Fig. 6(b) with the quasistatic axial magnetic field $B_{x}$ displayed in Fig. 7. The area where the magnetic field is maximal $\left(50 c / \omega_{0}<x<60 c / \omega_{0}\right)$ corresponds to the area where the electron $L_{x}$ values are optimal and where its associated transverse distribution is narrow. As this distribution transverse size increases (from $x=60 c / \omega_{0}$ to $x=100 c / \omega_{0}$ ), the magnetic field amplitude decreases. These results clearly demonstrate that the laser to plasma OAM transfer enables generation of a quasistatic axial magnetic field.

The quasistatic magnetic field generation is a robust effect and this field slowly decays in time, as shown in Fig. 8, drawing the magnetic field 60 fs later. Its profile evolves in time due to the electrons longitudinal oscillation between

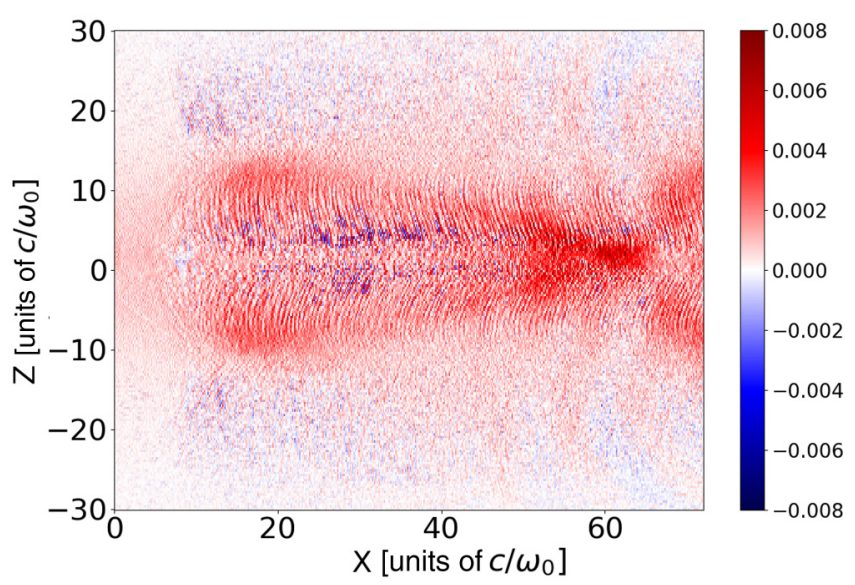

FIG. 7. Longitudinal $B_{x}$ field at $\omega_{0} t=165$, once the laser has left the plasma area, computed at $y_{0}=0$. Dashed line shows the left plasma boundary at $x=7.5 c / \omega_{0}$. The right boundary is at $x=$ $82.5 c / \omega_{0}$. the plasma edges. Indeed, in addition to the orbital angular momentum, the electrons gain also longitudinal momenta under the longitudinal ponderomotive force.

\section{CONCLUSION}

By using 3D particle in cell simulations, we model the interaction of a low dense plasma with relativistic LaguerreGauss beams characterized with three different polarizations: radial, linear, and azimuthal. We observe that the three polarizations lead to a laser to electron OAM transfer, but this transfer is more efficient in the case of radial polarization [9]. It appears to be an excellent candidate to efficiently generate an optimal "wireless" solenoid.

We develop a theoretical method to analytically describe the electromagnetic field components of Laguerre-Gaussian beams with any polarization. By inserting these expressions in the equations of motion, we analytically describe the dynamics of electrons irradiated by such laser beams in the perturbative regime. For linearly polarized LG beams, the perturbative theory predicts a laser to electron OAM transfer depending on the initial azimuthal position of electrons.

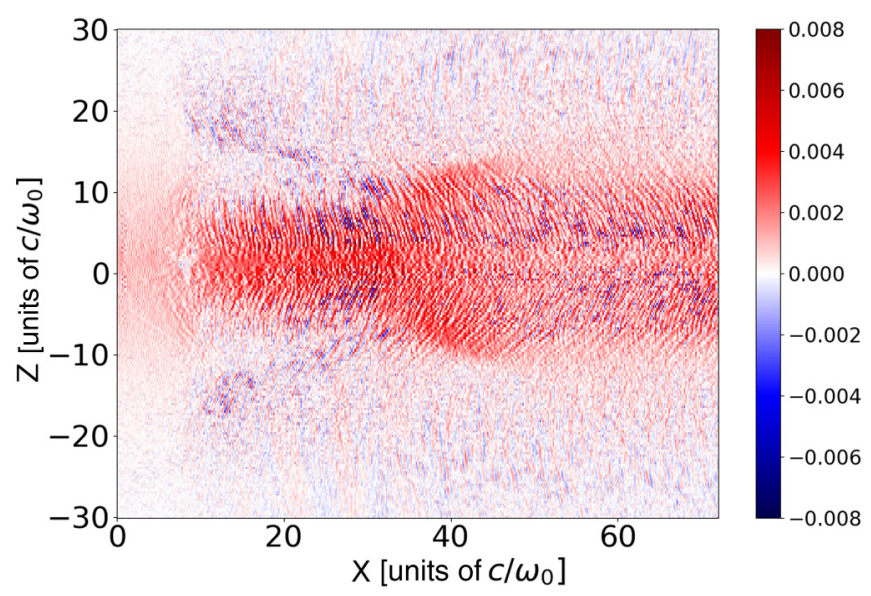

FIG. 8. Longitudinal $B_{x}$ field at $\omega_{0} t=315$ computed at $y_{0}=0$. Dashed line shows the left plasma boundary at $x=7.5 c / \omega_{0}$. The right boundary is at $x=82.5 \mathrm{c} / \omega_{0}$. 
Averaging the electron OAM values over all plasma particles should result in a zero plasma OAM. However, relativistic PIC simulations display a nonzero plasma OAM and the generation of a quasistatic magnetic field. The difference between the analytical expression and the numerical results originates from the forced radial oscillation of electrons combined with the longitudinal ponderomotive force which breaks the phase between the laser and the electrons. These processes are not included in the second order of the perturbative theory, and require additional analysis.

The physical processes responsible for the laser to plasma OAM transfer are similar to the one involved in the direct laser acceleration mechanism. Here, the constant part of the longitudinal ponderomotive force acts as a dephasing process as it increases the electron velocity. As a result, the electric fields seen by the electron are temporally chirped, and then electrons gain angular momentum in the forced radial oscillation in the laser.

\section{ACKNOWLEDGMENTS}

We acknowledge PRACE for awarding us access to JOLIOT CURIE at GENCI@CEA, France. The authors also acknowledge support from MEPhI Academic Excellence Project (Contract No. 02.a03.21.0005-27.08.2013).

\section{APPENDIX A: DIMENSIONLESS UNITS}

Throughout the manuscript, we express the physical quantities in dimensionless units. The mass and charge are expressed in electron mass $m_{e}$ and charge $e$, respectively. The velocities and momenta are expressed in $c$ and $m_{e} c$, respectively, where $c$ is the light velocity in vacuum. Time and lengths are expressed in $1 / \omega_{0}$ and $c / \omega_{0}$, respectively, with $\omega_{0}$ being the laser pulsation. Orbital angular momenta are expressed in $m_{e} c^{2} / \omega_{0}$. Electric and magnetic fields are expressed in $m_{e} \omega_{0} c / e$ and $m_{e} \omega_{0} / e$, respectively.

\section{APPENDIX B: DECOMPOSITION OF THE FIELDS OVER THE LG BEAMS BASIS}

Theoretical description of the electron dynamics requires the analytical expressions of the electromagnetic field components. Only the real part of the field components is considered in the electron dynamics equations. However, here, the decomposition procedure is general and considers the complex expressions for the field components.

Thus we expand the field components given by Eq. (1) over the orthonormal basis formed with the LG functions [10]:

$$
\begin{aligned}
U_{p, l}(r, \theta, t, x)= & \frac{C_{p, l}}{w(x)}\left(\frac{r \sqrt{2}}{w(x)}\right)^{|l|} L_{p}^{|l|}\left(\frac{2 r^{2}}{w^{2}(x)}\right) e^{-\left(\frac{r}{w(x)}\right)^{2}} e^{i l \theta} \\
& \times e^{i k \frac{x 2^{2}}{2\left(x^{2}+x_{R}^{2}\right)}} e^{-i(2 p+|l|+1) \arctan \left(\frac{x}{x_{R}}\right)}
\end{aligned}
$$

with the normalization factor $C_{p, l}=\sqrt{\frac{2}{\pi}} \sqrt{\frac{p !}{(p+|l|) !}}$, the Rayleigh length $x_{R}=\pi w_{0}^{2} / \lambda_{0}$, and the laser beam waist $w(x)=w_{0} \sqrt{1+\left(x / x_{R}\right)^{2}} . L_{p}^{l}(X)$ denotes the generalized Laguerre polynomials where the radial and azimuthal indexes are $p$ and $l$, respectively.

To simplify the computation, we consider $g(t)=1$ in Eq. (1) and write the electric field $y$ component as

$$
E_{y}(r, \theta, t, x)=\sum_{p^{\prime}, l^{\prime}} A_{y}^{p^{\prime}, l^{\prime}} U_{p^{\prime}, l^{\prime}}\left(r, \theta, t, x-x_{f}\right),
$$

with $A_{y}^{p^{\prime}, l^{\prime}}=\left\langle U_{p^{\prime}, l^{\prime}} \mid E_{y}\right\rangle=E_{0} C_{0, l} \frac{\pi w_{0}}{2^{1+\frac{l l}{2}}} \delta_{l^{\prime}, l} \delta_{p^{\prime}, 0}$. Figure 9 compares the real part of $E_{y}$ computed from Maxwell's consistent algorithm [14] and from Eq. (B2) for $l=1$; both expressions are shown at the focal point $\left(x=x_{f}\right)$. Decomposition over the LG beams basis [Fig. 9(b)], computed from Eq. (B2), fits exactly Maxwell's consistent solution [Fig. 9(a)], even for the considered small beam waist. We retrieve the two lobes with opposite signs originated from the helicoidal field distribution for $l=1$.
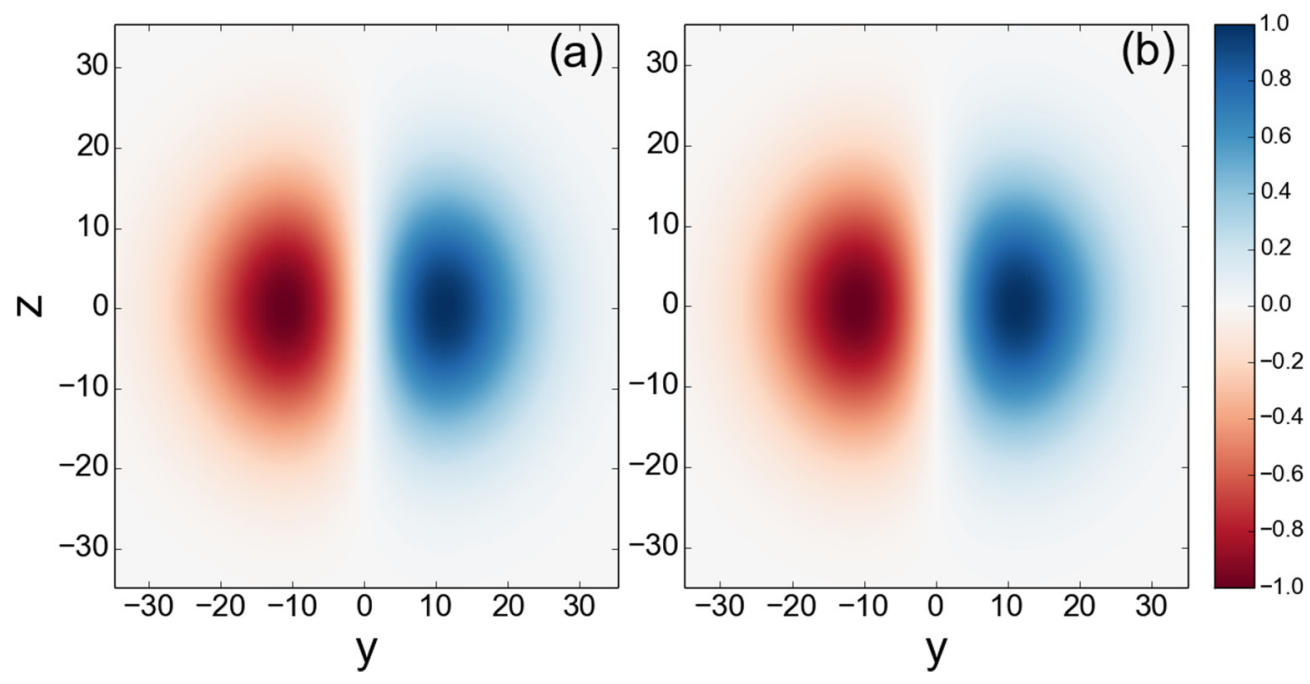

FIG. 9. Real part of the $E_{y}$ component computed at the focal point from Ref. [14] (a) and from the decomposition over the LG basis (b) for $l=1$ 

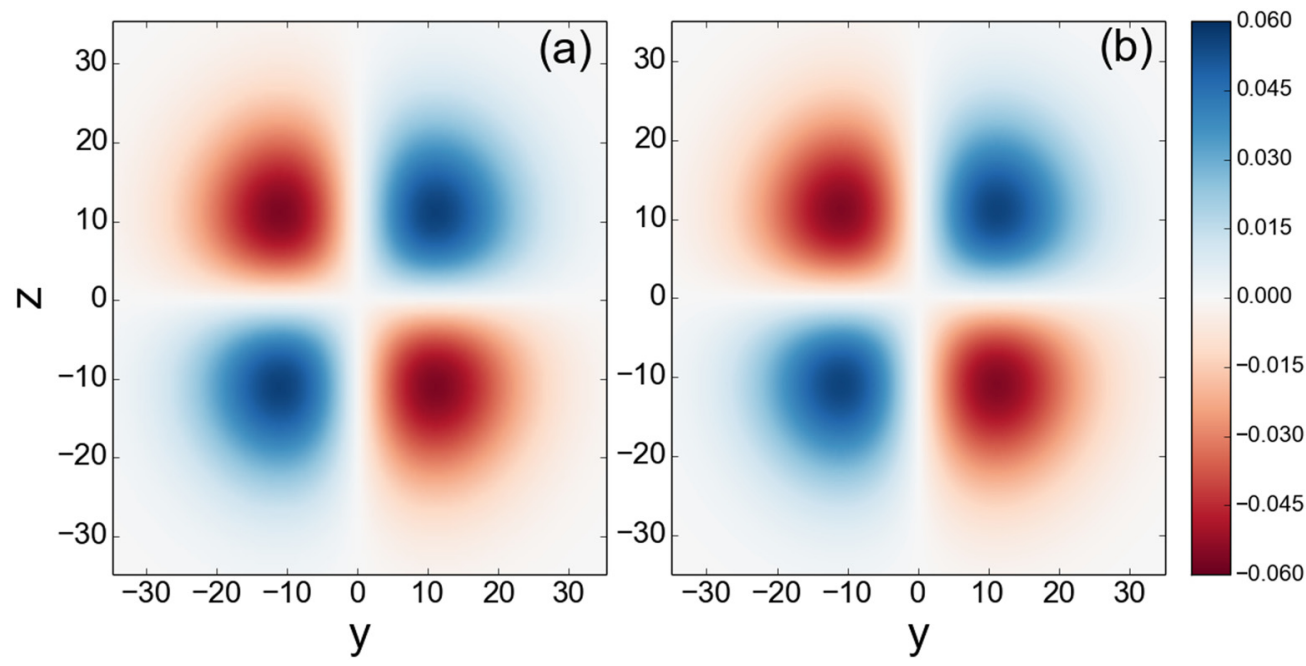

FIG. 10. Real part of the $E_{x}$ component computed at the focal point from Ref. [14] (a) and from the decomposition over the LG basis (b) for $l=1$.

We compute a longitudinal component of the electric field by solving the Poisson equation $\vec{\nabla} \vec{E}=0$ and assuming $E_{z}=0$ as

$$
\begin{aligned}
E_{x}(r, \theta, t, x) \simeq & i A_{y}^{0, l}\left[\frac{\sqrt{2}}{w\left(x-x_{f}\right)} \cos \theta U_{1,|l|-1} e^{i(l-|l|+1) \theta} e^{i(2+|| l|-1|-|l|) \arctan \frac{x-x_{f}}{x_{R}}}\right. \\
& \left.-2 \frac{r}{w^{2}\left(x-x_{f}\right)} \cos \theta \frac{1}{x_{R}}\left[1-i\left(x-x_{f}\right)\right] U_{0, l}\left(r, \theta, t, x-x_{f}\right)-i \frac{l}{r} \sin \theta U_{0, l}\left(r, \theta, t, x-x_{f}\right)\right],
\end{aligned}
$$

where we neglect a contribution related to the longitudinal variation of the Gouy phase. The real part of the $E_{x}$ components, computed from Maxwell's consistent algorithm and from the decomposition over the LG basis, are compared in Fig. 10. The longitudinal component involves a LG mode with an higher radial index, described by $U_{1,|l|-1}$ in Eq. (B3).

The magnetic field components, computed from the Maxwell-Faraday equation $\frac{\partial \vec{B}}{\partial t}=-\vec{\nabla} \times \vec{E}$, are $B_{z}=E_{y} / c, B_{y}=0$, and

$$
\begin{aligned}
B_{x}(r, \theta, t, x) \simeq & i A_{y}^{0, l}\left[\frac{\sqrt{2}}{w\left(x-x_{f}\right)} \sin \theta U_{1,|l|-1} e^{i(l-|l|+1) \theta} e^{i(2+|| l|-1|-|l|) \arctan \frac{x-x_{f}}{x_{R}}}\right. \\
& \left.+2 i \frac{r}{w^{2}\left(x-x_{f}\right)} \sin \theta \frac{x-x_{f}}{x_{R}} U_{0, l}\left(r, \theta, t, x-x_{f}\right)+i \frac{l}{r} \cos \theta U_{0, l}\left(r, \theta, t, x-x_{f}\right)\right] .
\end{aligned}
$$
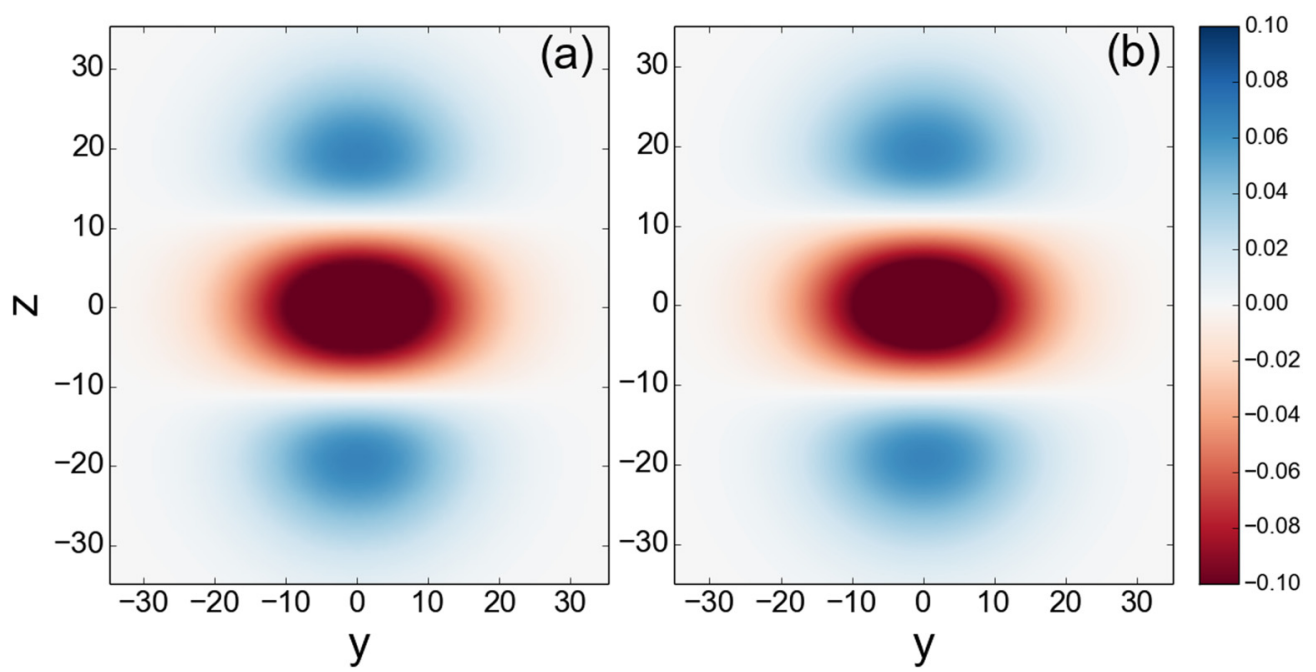

FIG. 11. Real part of the $B_{x}$ component computed at the focal point from Ref. [14] (a) and from the decomposition over the LG basis (b) for $l=1$. 
We present a comparison between the exact solution of $B_{x}$ and its decomposition over the LG beams basis in Fig. 11. As in the previous cases, we observe a perfect agreement between both panels. This agreement is also observed for both real and imaginary components (not shown here) computed in the whole space and, particularly, for the injection plane $\left(x=x_{b}\right)$. This method applies for electromagnetic fields which slightly diverge from the paraxial approximation, as the one presented in this article. Within a certain accuracy, it can be applied for all such beams carrying OAM with any polarization, such as, for example, radial or azimuthal polarization. It only requires an analytical expression of the transverse electric fields at the focal plane.

\section{APPENDIX C: PHYSICAL QUANTITIES AT THE FIRST ORDER}

The solutions of Eq. (15) are

$$
\begin{aligned}
& p_{r}^{(1)}(t)=-\frac{a_{0}}{2} f\left(r_{0}\right) \cos \theta_{0}\left[\sin \left(t-\phi_{0}\right)+\frac{1}{2\left(1+\frac{1}{n}\right)} \sin \left(\left[1+\frac{1}{n}\right] t-\phi_{0}\right)\right. \\
& \left.+\frac{1}{2\left(1-\frac{1}{n}\right)} \sin \left(\left[1-\frac{1}{n}\right] t-\phi_{0}\right)-(-1)^{n} \frac{1}{n^{2}-1} \sin \phi_{0}\right], \\
& r^{(1)}(t)=\frac{a_{0}}{2} f\left(r_{0}\right) \cos \theta_{0}\left[\cos \left(t-\phi_{0}\right)+\frac{1}{2\left(1+\frac{1}{n}\right)^{2}} \cos \left(\left[1+\frac{1}{n}\right] t-\phi_{0}\right)\right. \\
& \left.+\frac{1}{2\left(1-\frac{1}{n}\right)^{2}} \cos \left(\left[1-\frac{1}{n}\right] t-\phi_{0}\right)+(-1)^{n} \frac{3 n^{2}-1}{\left(n^{2}-1\right)^{2}} \cos \phi_{0}+(-1)^{n} \frac{n \pi+t}{n^{2}-1} \sin \phi_{0}\right], \\
& p_{\theta}^{(1)}(t)=\frac{a_{0}}{2} f\left(r_{0}\right) \sin \theta_{0}\left[\sin \left(t-\phi_{0}\right)+\frac{1}{2\left(1+\frac{1}{n}\right)} \sin \left(\left[1+\frac{1}{n}\right] t-\phi_{0}\right)\right. \\
& \left.+\frac{1}{2\left(1-\frac{1}{n}\right)} \sin \left(\left[1-\frac{1}{n}\right] t-\phi_{0}\right)-(-1)^{n} \frac{1}{n^{2}-1} \sin \phi_{0}\right], \\
& \theta^{(1)}(t)=-\frac{a_{0}}{2} \frac{f\left(r_{0}\right)}{r_{0}} \sin \theta_{0}\left[\cos \left(t-\phi_{0}\right)+\frac{1}{2\left(1+\frac{1}{n}\right)^{2}} \cos \left(\left[1+\frac{1}{n}\right] t-\phi_{0}\right)\right. \\
& \left.+\frac{1}{2\left(1-\frac{1}{n}\right)^{2}} \cos \left(\left[1-\frac{1}{n}\right] t-\phi_{0}\right)+(-1)^{n} \frac{3 n^{2}-1}{\left(n^{2}-1\right)^{2}} \cos \phi_{0}+(-1)^{n} \frac{t+n \pi}{n^{2}-1} \sin \phi_{0}\right], \\
& p_{x}^{(1)}(t)=-\frac{a_{0}}{2} \frac{f\left(r_{0}\right)}{r_{0}}\left\{-\left[|l|-2\left(\frac{r_{0}}{w_{0}}\right)^{2}\right] \cos \theta_{0}\left[\cos \left(t-\phi_{0}\right)+\frac{1}{2\left(1+\frac{1}{n}\right)} \cos \left(\left[1+\frac{1}{n}\right] t-\phi_{0}\right)\right.\right. \\
& \left.+\frac{1}{2\left(1-\frac{1}{n}\right)} \cos \left(\left[1-\frac{1}{n}\right] t-\phi_{0}\right)+(-1)^{n} \frac{1}{n^{2}-1} \cos \phi_{0}\right]+l \sin \theta_{0}\left[\sin \left(t-\phi_{0}\right)+\frac{1}{2\left(1+\frac{1}{n}\right)} \sin \left(\left[1+\frac{1}{n}\right] t-\phi_{0}\right)\right. \\
& \left.\left.+\frac{1}{2\left(1-\frac{1}{n}\right)} \sin \left(\left[1-\frac{1}{n}\right] t-\phi_{0}\right)-\frac{(-1)^{n}}{n^{2}-1} \sin \phi_{0}\right]\right\} \\
& x^{(1)}(t)=\frac{a_{0}}{2} \frac{f\left(r_{0}\right)}{r_{0}}\left\{[ | l | - 2 ( \frac { r _ { 0 } } { w _ { 0 } } ) ^ { 2 } ] \operatorname { c o s } \theta _ { 0 } \left[\sin \left(t-\phi_{0}\right)+\frac{1}{2\left(1+\frac{1}{n}\right)^{2}} \sin \left(\left[1+\frac{1}{n}\right] t-\phi_{0}\right)\right.\right. \\
& \left.+\frac{1}{2\left(1-\frac{1}{n}\right)^{2}} \sin \left(\left[1-\frac{1}{n}\right] t-\phi_{0}\right)-(-1)^{n} \frac{3 n^{2}-1}{\left(n^{2}-1\right)^{2}} \sin \phi_{0}+(-1)^{n} \frac{t+n \pi}{n^{2}-1} \cos \phi_{0}\right] \\
& +l \sin \theta_{0}\left[\cos \left(t-\phi_{0}\right)+\frac{1}{2\left(1+\frac{1}{n}\right)^{2}} \cos \left(\left[1+\frac{1}{n}\right] t-\phi_{0}\right)+\frac{1}{2\left(1-\frac{1}{n}\right)^{2}} \cos \left(\left[1-\frac{1}{n}\right] t-\phi_{0}\right)\right. \\
& \left.\left.+(-1)^{n} \frac{3 n^{2}-1}{\left(n^{2}-1\right)^{2}} \cos \phi_{0}+(-1)^{n} \frac{t+n \pi}{n^{2}-1} \sin \phi_{0}\right]\right\} \text {. }
\end{aligned}
$$

[1] G. Gariepy, J. Leach, K. T. Kim, T. J. Hammond, E. Frumker, R. W. Boyd, and P. B. Corkum, Creating High-Harminc Beams with Controlled Orbital Angular Momentum, Phys. Rev. Lett. 113, 153901 (2014).

[2] J. Viera, R. M. G. M. Trines, E. P. Alves, R. A. Fonseca, J. T. Mendonça, R. Bingham, P. Norreys, and L. O. Silva, High
Orbital Angular Momentum Harmonic Generation, Phys. Rev. Lett. 117, 265001 (2016).

[3] J. Viera, R. M. G. M. Trines, E. P. Alves, R. A. Fonseca, J. T. Mendonça, R. Bingham, P. Norreys, and L. O. Silva, Amplification and generation of ultra-intense twisted laser pulses via stimulated raman scattering, Nat. Commun. 7, 10371 (2016). 
[4] J. T. Mendonça, B. Thidé, and H. Then, Stimulated Raman and Brillouin Backscattering of Collimated Beams Carrying Orbital Angular Momentum, Phys. Rev. Lett. 102, 185005 (2009).

[5] J. Viera and J. T. Mendonça, Nonlinear Laser Driven Donut Wakefields for Positron and Electron Acceleration, Phys. Rev. Lett. 112, 215001 (2014).

[6] S. Ali, J. R. Davies, and J. T. Mendonça, Inverse Faraday Effect with Linearly Polarized Laser Pulses, Phys. Rev. Lett. 105, 035001 (2010).

[7] Y. Shi, J. Viera, R. M. G. M. Trines, R. Bingham, B. F. Shen, and R. J. Kingham, Magnetic Field Generation in Plasma Waves Driven by Copropagating Intense Twisted Lasers, Phys. Rev. Lett. 121, 145002 (2018).

[8] L.-X. hu, T.-P. Yu, Y. Lu, g. B. Zhang, D.-B. Zou, H. Zhang, Z.-Y. Ge, Y. Yin, and F.-Q. Shao, Dynamics of the interaction of relativisitc Laguerre-Gaussian laser pulses with a wire target, Plasma Phys. Control Fusion 61, 025009 (2019).

[9] R. Nuter, Ph. Korneev, I. Thiele, and V. T. Tikhonchuk, Plasma solenoid driven by a laser beam carrying an orbital angular momentum, Phys. Rev. E 98, 033211 (2018).

[10] L. Allen, M. W. Beijersbergen, R. J. C. Spreeuw, and J. P. Woerdman, Orbital angular momentum of light and the transformation of Laguerre-Gaussian laser modes, Phys. Rev. A 45, 8185 (1992).
[11] A. Pukhov, 3d electromagnetic relativistic particle-in-cell code VLPL: Virtual laser plasma laboratory, J. Plasma Phys. 61, 425 (1999).

[12] A. V. Arefiev, B. N. Breizman, M. Schollmeier, and V. N. Khudik, Parametric Amplification of Laser-Driven Electron Acceleration in Underdense Plasma, Phys. Rev. Lett. 108, 145004 (2012).

[13] R. Nuter and V. T. Tikhonchuk, Prepulse suppression and optimization of backward Raman amplification with a chirped pump laser beam, Phys. Rev. E 87, 043109 (2013).

[14] I. Thiele, S. Skupin, and R. Nuter, Boundary conditions for arbitrarily shaped and tightly focused laser pulses in electromagnetic codes, J. Comput. Phys. 321, 1110 (2016).

[15] A. P. L. Robinson, A. V. Areviev, and D. Neely, Generating "Superponderomotive" Electrons Due to a Non-Wake-Field Interaction between a Laser Pulse and a Longitudinal Electric Field, Phys. Rev. Lett. 111, 065002 (2013).

[16] R. Zhang, L.-H. Cheng, R.-A. Tang, and J.-K. Xue, Direct laser acceleration in an inhomogeneous cylindrical plasma channel, Phys. Plasmas 23, 093105 (2016).

[17] P. Mora, Plasma Expansion into a Vacuum, Phys. Rev. Lett. 90, 185002 (2003). 